\title{
Dynamics of APRI and FIB-4 in HCV cirrhotic patients who achieved SVR after DAA therapy
}

\author{
ANCA LEUŞTEAN ${ }^{1,2}$, CRISTINA POPESCU ${ }^{1,2}$, LUCIANA NICHITA $^{3,4}$, \\ CĂTĂLIN TILIŞCAN ${ }^{2,5}$ and VICTORIA ARAMÄ $\breve{~}^{1,2}$
}

\begin{abstract}
${ }^{1}$ Department of Infectious Diseases, Faculty of Medicine, 'Carol Davila' University of Medicine and Pharmacy, 020021 Bucharest; ${ }^{2}$ Third Clinical Department, 'Prof. Dr. Matei Balş' National Institute for Infectious Diseases, 021105 Bucharest; ${ }^{3}$ Department of Pathology, Faculty of Dental Medicine, 'Carol Davila' University of Medicine and Pharmacy, 020021 Bucharest; ${ }^{4}$ Department of Pathology, Colentina University Hospital, 020125 Bucharest; ${ }^{5}$ Department of Pathophysiology and Immunology, Faculty of Dental Medicine, ‘Carol Davila' University of Medicine and Pharmacy, 020021 Bucharest, Romania
\end{abstract}

Received August 26, 2020; Accepted September 25, 2020

DOI: $10.3892 /$ etm.2020.9531

\begin{abstract}
There are limited data available on the regression of fibrosis in hepatitis $\mathrm{C}$ virus (HCV) patients who have achieved sustained virologic response (SVR) after interferon-free treatments. Moreover, a perfect method for assessing liver fibrosis and its dynamics has not been established yet. The main objective of this study was to evaluate the dynamics of aspartate aminotransferase to platelet ratio index (APRI) and Fibrosis-4 (FIB-4) scores in patients with HCV who registered SVR. We performed ROC curve analysis to evaluate the diagnostic performance of APRI and FIB-4 scores in determining the presence of cirrhosis in comparison to FibroTest. In total 251 patients were enrolled: 164 cirrhotic and 83
\end{abstract}

Correspondence to: Dr Anca Leuştean, Department of Infectious Diseases, Faculty of Medicine, 'Carol Davila' University of Medicine and Pharmacy, 37 Dionisie Lupu Street, Sector 1, 020021 Bucharest, Romania

E-mail: anca_leustean@yahoo.com

Abbreviations: ALT, alanine aminotransferase; APRI, aspartate aminotransferase to platelet ratio index; AST, aspartate aminotransferase; AUROC, area under the receiver operating characteristics curve; BMI, body mass index; CBC, complete blood count; CI, confidence interval; DAA, direct acting antivirals; ECM, extracellular matrix; EOT, end of treatment; FIB-4, Fibrosis-4 score; GGT, gamma-glutamyl transferase; HA, hyaluronic acid; HCC, hepatocellular carcinoma; HCV, hepatitis C virus; HDL, high-density lipoprotein; HOMA-IR, homeostatic model assessment insulin resistance; HSC, hepatic stellate cells; IU, international units; LB, liver biopsy; NPV, negative predictive value; PPV, positive predictive value; SD, standard deviation; SVR, sustained virologic response; TE, transient elastography

Key words: APRI, FIB-4, HCV cirrhosis, DAA therapy, sustained virologic response non-cirrhotic patients, and they were evaluated at baseline, at 6 and at 12 months post-end of treatment (EOT). In the cirrhotic group, at baseline, there was a weak but statistically significant correlation between APRI and FibroTest $(\tau=0.173$, $\mathrm{P}=0.001)$, as well as between FIB-4 and FibroTest $(\tau=0.265$, $\mathrm{P}<0.001)$. At the 6-month follow-up, APRI no longer correlated with FibroTest $(\tau=0.144, \mathrm{P}=0.057)$, while FIB-4 was correlated $(\tau=0.256, P=0.001)$. The same pattern was shown at 12 months post-EOT. Between baseline and the 6-month evaluation, there was a significant decrease in APRI $(\mathrm{P}<0.001)$ and FIB-4 $(\mathrm{P}<0.001)$ scores, but for the next follow-up period, there was no reduction. In the non-cirrhotic group, APRI and FIB-4 did not correlate with the FibroTest value at any of the evaluation times. There was a significant difference between baseline and the 6 -month visit for APRI $(\mathrm{P}=0.01)$ and for FIB-4 $(\mathrm{P}=0.014)$. The areas under the receiver operating characteristics curve (AUROCs) for the presence of cirrhosis compared with FibroTest for APRI and FIB-4 were 0.682 [95\% confidence interval (CI), 0.613-0.752] and 0.693 (95\% CI 0.625-0.76). Both APRI and FIB-4 prove to be easy, quick and inexpensive tools for screening $\mathrm{HCV}$ cirrhosis, with moderate diagnostic accuracy and FIB-4 can be useful for monitoring patients post-EOT.

\section{Introduction}

Since November 2015, direct-acting antiviral (DAA) based regimens have been used in Romania for treating patients with hepatitis $\mathrm{C}$ virus ( $\mathrm{HCV}$ ) infection. The patients with liver cirrhosis were treated during the first year that the protocol was being implemented in Romania $(1,2)$ and in the following years non-cirrhotic patients were also included. The percentage of sustained virologic response (SVR) in patients with HCV genotype 1 (found in $99 \%$ of the $\mathrm{HCV}$ infected patients in Romania) is known to be over 95\% (3). There are limited previous data collected on the regression of fibrosis in patients who have achieved SVR after interferon-free treatments. A perfect method for assessing liver fibrosis and 
its dynamics has not been established yet. The gold standard for evaluating the liver fibrosis has been the liver biopsy (LB), but in the recent years, non-invasive methods, especially the FibroTest, have been used instead (4). However, liver biopsy has significant limitations: possible complications during the procedure, sometimes with life-threatening potential (5), difficulties to carry out serial examinations in order to monitor the dynamic of liver fibrosis, some patients may be afraid to do the test, and usually the test is poorly accepted. Small liver samples may not always be sufficient to estimate the structure of such a large organ (sampling errors because it only evaluates $1 / 50,000$ of the liver parenchyma). Moreover, it is considered that fibrosis has a heterogeneous disposition in the liver (6-8). The optimal size of liver fragment at liver biopsy seems to be around $40 \mathrm{~mm}$ and the acceptable size is $25 \mathrm{~mm}$. However, it is difficult to obtain optimal size fragments and Poynard et al (9) analyzed in 2004 more than 10,000 liver biopsies with 25-35\% inadequate sample size.

Histopathological aspects from different fibrosis stages of the liver tissue in patients with chronic hepatitis obtained through liver biopsy are shown in Fig. 1. Using Masson staining of liver tissue, stage 1 Ishak fibrosis score (A) corresponds to fibrous expansion of portal tracts with inflammatory cell infiltration; stage 3 Ishak fibrosis score (B) consists in fibrous septa which form occasional bridges between adjacent vascular structures; stage 5 Ishak fibrosis score (C) appears later in the progression of disease, with numerous bridges and rare parenchymal nodules completely surrounded by fibrosis; the late stage: Stage 6 Ishak score-cirrhosis (D) corresponds to the entire tissue being composed of parenchymal nodules surrounded by fibrosis (Fig. 1).

In Romania, the national guidelines use the FibroTest as a reference. Other non-invasive, cheaper and faster methods for evaluating the liver fibrosis in HCV infected patients have been developed by scientists, among them being the aspartate aminotransferase to platelet ratio index (APRI) (10) and the Fibrosis-4 (FIB-4) score (11), but these are rarely used in daily practice.

A meta-analysis conducted in 2018 by Zubair and Wajid (12) stresses upon the fact that the FibroTest (although not a perfect method), has a better diagnostic accuracy that APRI and FIB-4, but it is more expensive, not as accessible and as simple as calculating the APRI and FIB-4 scores.

The main objective of this study was to evaluate the dynamics of APRI and FIB-4 scores in patients with HCV who registered SVR and to evaluate if they could be useful and less expensive tools for screening $\mathrm{HCV}$ patients for cirrhosis and monitoring after DAA treatment. We performed ROC curve analysis to evaluate the diagnostic performance of APRI and FIB-4 scores in determining the presence of cirrhosis in comparison to FibroTest.

\section{Materials and methods}

Design and ethics. This study is a prospective observational analysis of $\mathrm{HCV}$ patients, both cirrhotic and non-cirrhotic (liver fibrosis determined by FibroTest), treated with DAA therapies and monitored in a tertiary-care infectious disease hospital, 'Prof. Dr. Matei Balş' National Institute for Infectious Diseases (Bucharest, Romania). The study was approved by the Ethics Committee and all patients signed an informed consent before inclusion in the analysis.

Patients were enrolled between November 2015 and January 2020 and included HCV patients who received DAA therapies for 12 weeks and achieved SVR. According to the National Protocol, patients were categorized as cirrhotic by having a result at FibroTest of F3-F4 or F4, each level below and equal to F3 being considered non-cirrhotic. All the patients included in the study were evaluated at baseline (before the start of treatment), at the first visit, at 6-months after the end of treatment (post-EOT), the 2nd visit and at 12 -months post-EOT, the 3rd visit. At each visit, FibroTest, APRI and FIB-4 scores were determined for each patient. The main group of study consisted of cirrhotic patients (164 patients), as labeled by the National Protocol using the scores of FibroTest of F3-F4 or F4 (a value of $>0.72$ ) and a control group of $\mathrm{HCV}$ non-cirrhotic patients (83 patients $\leq$ F3).

APRI was calculated with the formula: [AST (IU/1)/AST (Upper Limit of Normal-IU/1)/Platelet count (10\%/1)] x100 and the patients were distributed according to prior determined cut-offs from the medical literature $(<1,1-2,>2)(13)$. FIB-4 was determined according to the formula: [Age (years) $\mathrm{x}$ AST level (IU/1)]/[(Platelet count $\left(10^{9} / 1\right) \times \sqrt{ }$ ALT(IU/1)] and patients were distributed by previously studied cut-offs $(<1.45,1.45-3.25,>3.25)(14)$.

Information was gathered regarding the demographic parameters (age, gender) and complete medical history for all patients, and at each visit we determined FibroTest and biological parameters: including complete blood count (CBC), complete biochemistry analysis and coagulation parameters.

Statistical analysis. The statistical analysis was performed using IBM SPSS ${ }^{\circledR}$ Statistics version 22 (IBM Corp.). In univariate analysis, the type of variable distribution was assessed by visual inspection of histograms, Q-Q plots and the Shapiro-Wilk test. The central tendency and dispersion for non-Gaussian distributed variables were expressed as median and interquartile range (IQR). In multivariate analysis, associations between continuous non-Gaussian distributed variables were assessed using Kendall's tau-b ( $\tau b$ ) correlation coefficient. Paired t-test was used to determine the significance of differences between paired continuous sample data. The Friedman test was also performed for differences between three groups of continuous data that has marked deviations from normality. ROC curve analysis was performed to assess the diagnostic ability of several variables. $\mathrm{P}<0.05$ was considered to indicate a statistically significant difference.

\section{Results}

A total of 251 patients were enrolled in the study, divided into 2 groups: The cirrhotic patients (164 patients) and the non-cirrhotic patients (83 patients). The median age for the cirrhotic group was 62.5 years (35-80); 92 were females $(56.1 \%)$ and $72(43.9 \%)$ were males. The median age of the non-cirrhotic group was 63 years (33-85), including 57 (68.7\%) females and $26(31.3 \%)$ males.

At baseline, FibroTest, APRI and FIB-4 scores were performed for both groups and the results are shown in Table I. 
Table I. Baseline APRI, FIB-4 and FibroTest scores in cirrhosis vs. non-cirrhosis groups.

\begin{tabular}{|c|c|c|c|}
\hline Variable & Cirrhosis (164 patients) (F3-F4 and F4) & Non-cirrhosis (83 patients) (F0-F3) & P-value \\
\hline \multicolumn{4}{|l|}{ APRI $(n, \%)$} \\
\hline Median (IQR) & $1.14(0.72-2.3)$ & $0.73(0.41-1.13)$ & 0.002 \\
\hline$<1$ & $67(40.85)$ & $58(69.88)$ & \\
\hline $1-2$ & $47(28.66)$ & $16(19.28)$ & \\
\hline$>2$ & $50(30.49)$ & $9(10.84)$ & \\
\hline \multicolumn{4}{|l|}{ FIB-4 (n, \%) } \\
\hline Median (IQR) & $3.32(2.17-5.32)$ & $2.21(1.5-2.98)$ & $<0.0001$ \\
\hline$<1.35$ & $13(7.93)$ & $11(13.25)$ & \\
\hline $1.35-3.25$ & $75(45.73)$ & $55(66.27)$ & \\
\hline$>3.25$ & $86(52.44)$ & $17(20.48)$ & \\
\hline \multicolumn{4}{|l|}{ FibroTest } \\
\hline Median (IQR) & $0.84(0.78-0.9)$ & $0.63(0.58-0.68)$ & $<0.0001$ \\
\hline
\end{tabular}
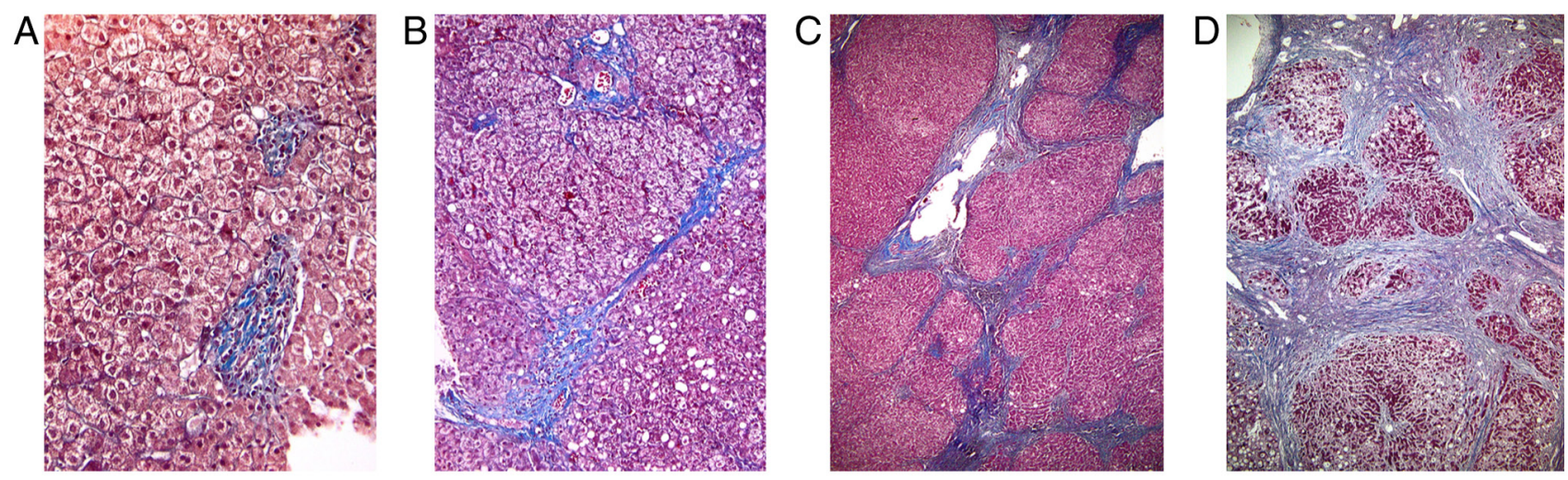

Figure 1. Masson staining of liver tissue with chronic hepatitis. (A) Fibrous expansion of portal tracts with inflammatory cell infiltration; below short spike-like septa (stage 1 Ishak fibrosis score). Magnification, x200. (B) Fibrous septa form occasional bridges between adjacent vascular structures (stage 3 Ishak fibrosis score). Magnification, x100. (C) With progression of disease, numerous bridges are formed and rare parenchymal nodules completely surrounded by fibrosis may form (stage 5 Ishak fibrosis score). Magnification, x40. (D) The entire tissue is composed of parenchymal nodules surrounded by fibrosis (stage 6 Ishak score-cirrhosis). Magnification, $\mathrm{x} 40$.

For the second visit (6 months post-EOT) and the third visit (12 months post-EOT), the same evaluations were performed and are described in Table II (for the cirrhotic patients) and Table III (for the non-cirrhotic patients).

In the cirrhotic group, at baseline, correlations were made between the FibroTests, APRI and FIB-4 and the results showed that there was a weak, but statistically significant correlation between APRI and FibroTest $(\tau=0.173, \mathrm{P}=0.001)$, as well as between FIB- 4 and FibroTest $(\tau=0.265, P<0.001)$. At the 6 months follow-up, APRI no longer correlated with the FibroTest ( $\tau=0.144, \mathrm{P}=0.057)$, but the FIB-4 score had a weak correlation with the gold standard of the study ( $\tau=0.256, \mathrm{P}=0.001)$. The same pattern was observed at 12 months post-EOT, APRI did not correlate with FibroTest $(\tau=0.100, \mathrm{P}=0.255)$, but FIB-4 showed significant correlation $(\tau=0.200, \mathrm{P}=0.023)$.

For the cirrhotic patients group, Friedman tests were performed which showed that there was a statistically signifi- cant difference between the baseline and the two follow-up visits of APRI values $(\mathrm{P}<0.001)$, FIB-4 values $(\mathrm{P}<0.001)$ and FibroTest values $(\mathrm{P}<0.001)$. The study showed that between baseline and the 6-month evaluation, there was a statistically significant difference for APRI $(\mathrm{P}<0.001$, confidence interval (CI) 95\%: 0.982-1.61) and for FIB-4 (P<0.001,95\% CI, 1.43-2.26), but for the next follow-up period (between 6 and 12 months post-EOT) no reduction for these scores $(\mathrm{P}=0.739$ for APRI, P=0.913 for FIB-4) was observed.

On the contrary, in the non-cirrhotic group, APRI and FIB-4 did not correlate with the FibroTest at any of the evaluation times. For APRI the results were: Baseline: $\tau=0.015$, $\mathrm{P}=0.841$, visit $2: \tau=0.104, \mathrm{P}=0.402$, visit 3: $\tau=0.005, \mathrm{P}=0.974$; and for FIB-4: Baseline: $\tau=0.041, P=0.589$, visit $2: \tau=0.107$, $\mathrm{P}=0.384$, visit 3: $\tau=-0.037, \mathrm{P}=0.820$.

Friedman tests were performed for this group as well and they showed that there was a statistically significant differ- 


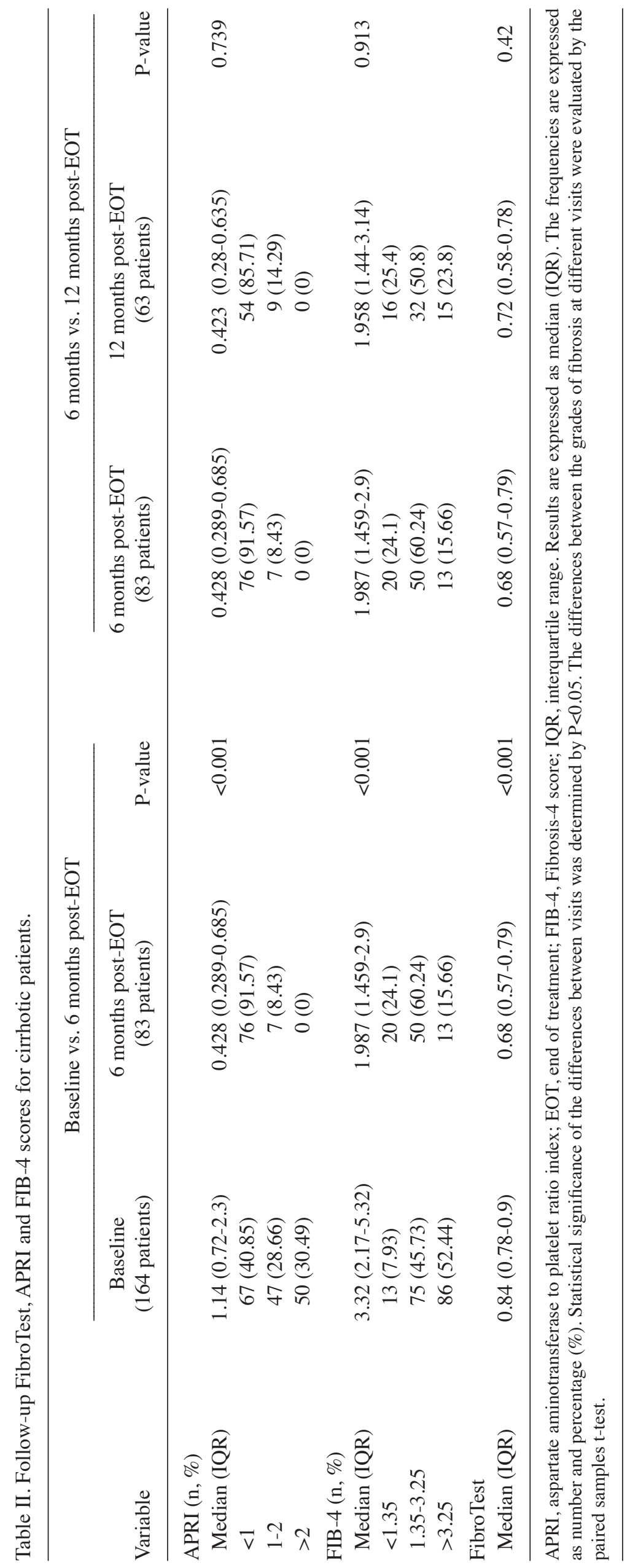




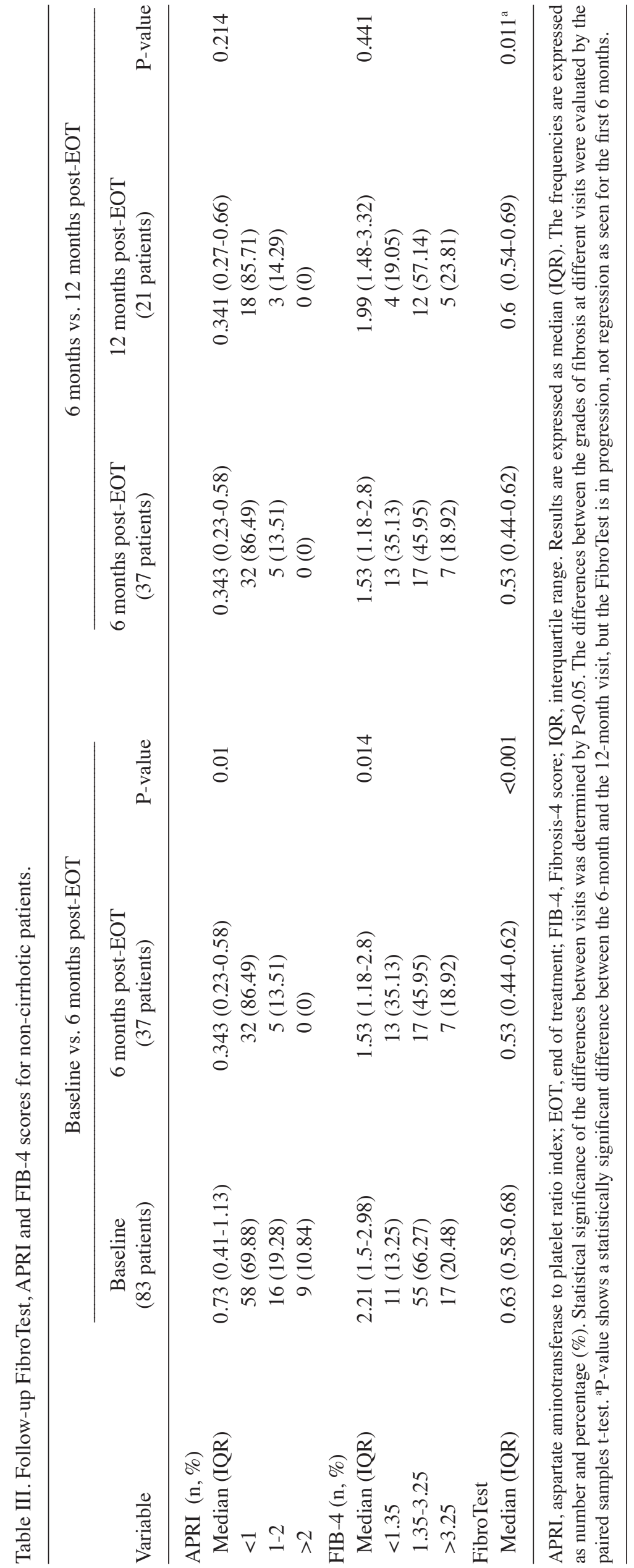




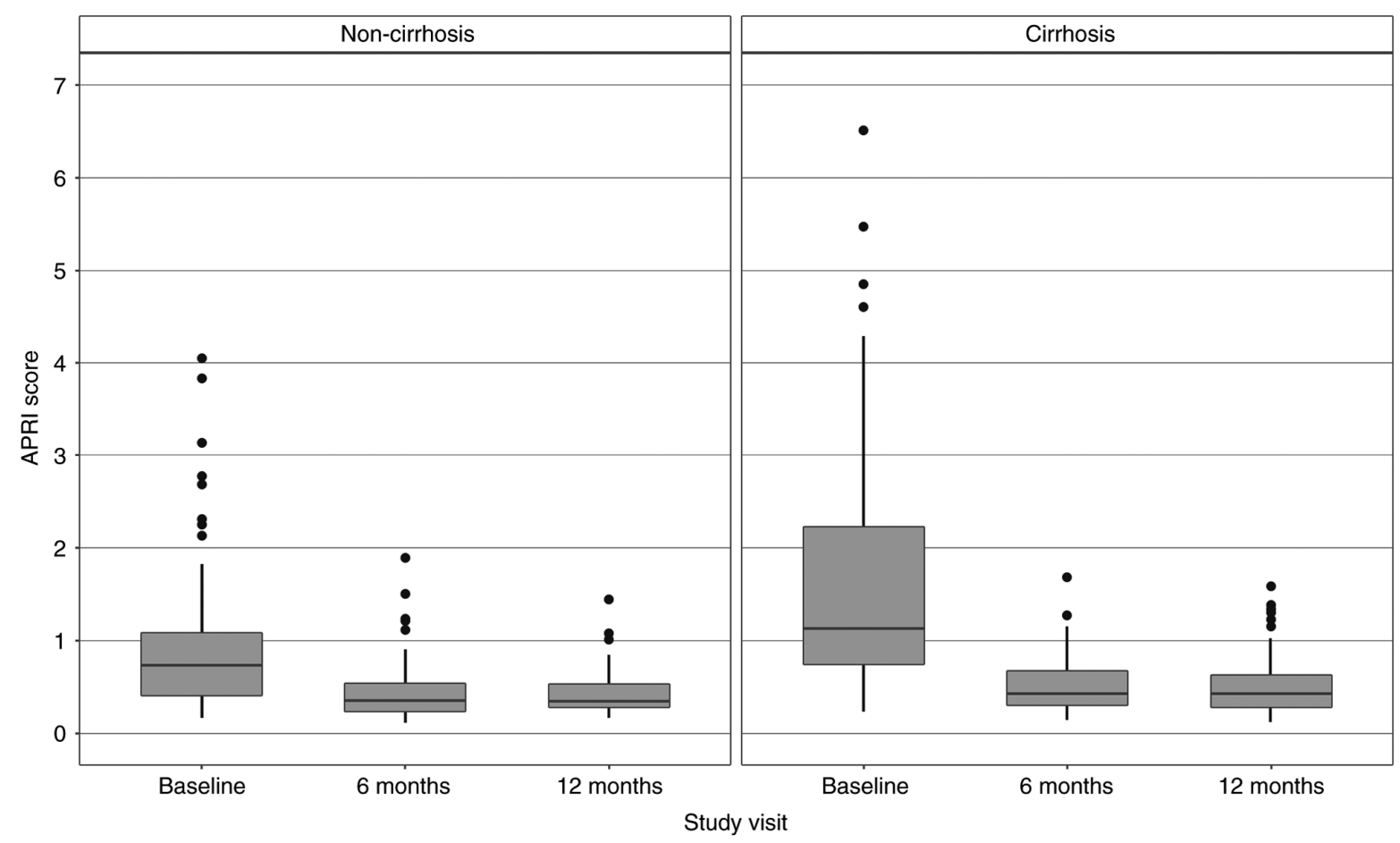

Figure 2. Regression of APRI score between baseline and 6 months and 12 months post-EOT in non-cirrhotic and cirrhotic HCV patients. APRI, aspartate aminotransferase to platelet ratio index; EOT, end of treatment; $\mathrm{HCV}$, hepatitis $\mathrm{C}$ virus.

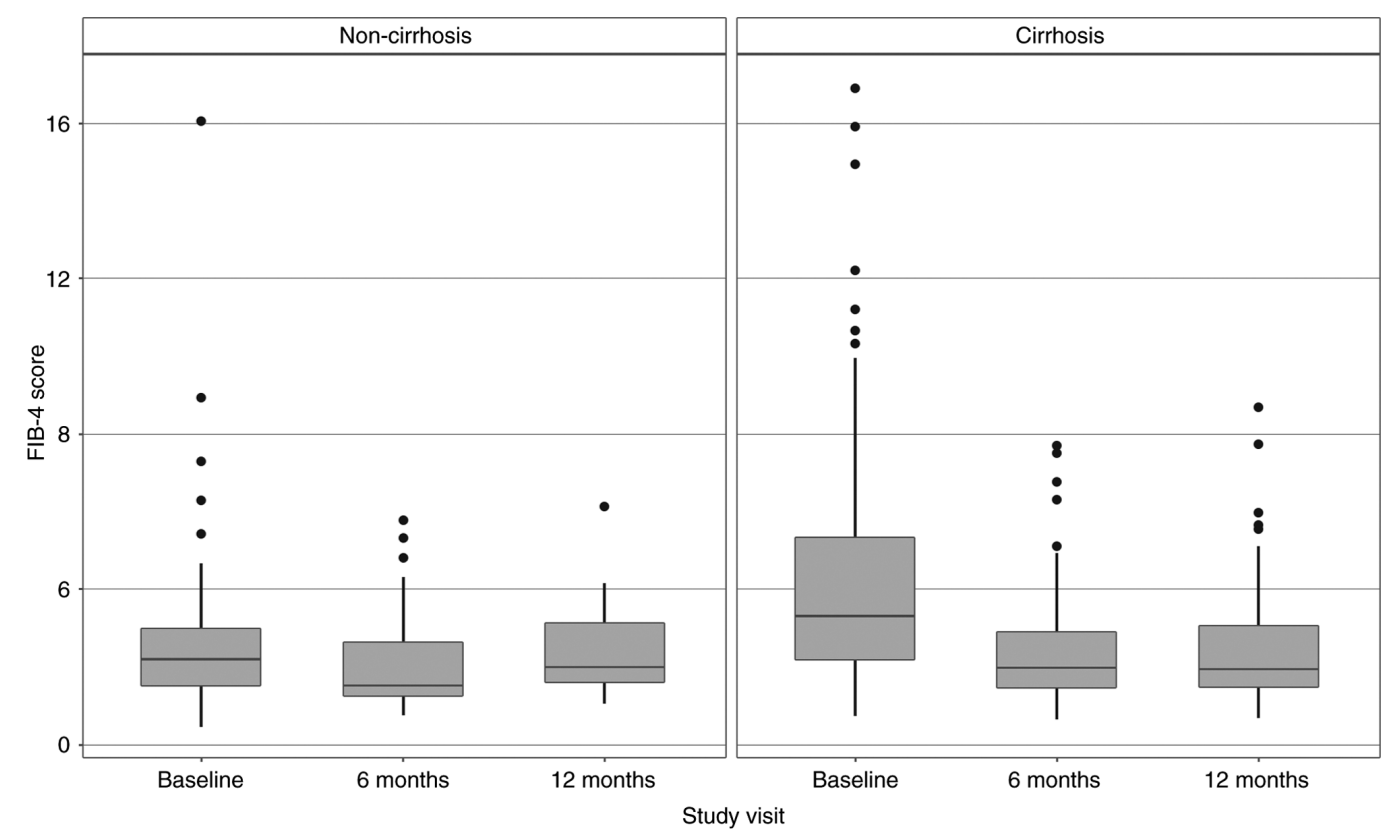

Figure 3. Regression of FIB-4 score between baseline and 6 months and 12 months post-EOT in non-cirrhotic and cirrhotic HCV patients. FIB-4, Fibrosis-4 score; EOT, end of treatment; HCV, hepatitis C virus.

ence between visits in the APRI values $(\mathrm{P}<0.001)$, FIB-4 values $(\mathrm{P}<0.001)$ and FibroTest values $(\mathrm{P}=0.02)$. However, the statistically significant difference was observed between baseline and the 6 -month visit $(\mathrm{P}=0.01$ for APRI and $\mathrm{P}=0.014$ for FIB-4), but for the next 6 months no reduction was shown.

The regression of APRI and FIB-4 scores in the cirrhotic and non-cirrhotic groups can also be observed in Fig. 2 (for APRI) and Fig. 3 (for FIB-4).
Additionally to these results, we performed the area under the receiver operating characteristics curve (AUROC), sensitivities, specificities, positive predictive value (PPV) and negative predictive value (NPV) for our data in evaluating the presence of cirrhosis in comparison to the FibroTest for the two scores: APRI and FIB-4 when differentiating F0-F3 vs. F4. The ROC curves for APRI and FIB-4 are presented in Fig. 4, and the AUROC of these 2 scores have 


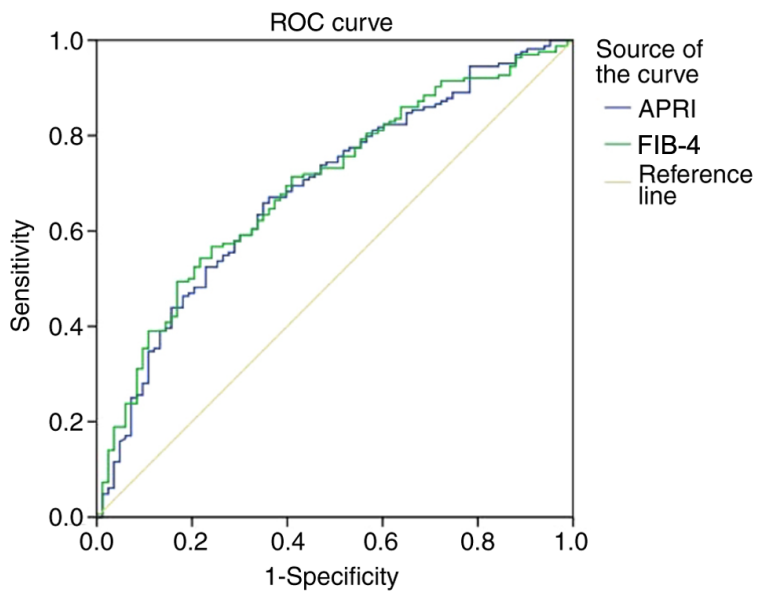

Figure 4. Receiver operating characteristic (ROC) curves for APRI and FIB-4 scores in differentiating F0F1F2F3 vs. F4. APRI, aspartate aminotransferase to platelet ratio index; FIB-4, Fibrosis-4 score.

values of $0.682(95 \% \mathrm{CI}, 0.613-0.752)$ for APRI and 0.693 (95\% CI, 0.625-0.76) for FIB-4 (Fig. 4).

The calculated optimal cut-off value for APRI was 0.867 and for this value, the score had a sensitivity of $68 \%$, a specificity of $58 \%$, a PPV of $76 \%$ and NPV of $48 \%$ for predicting cirrhosis (F4) in comparison to F0-F3. For the FIB-4 score, at a cut-off of 2.32 , the sensitivity was $71 \%$, the specificity was $58 \%$, the PPV was $76.9 \%$ and NPV was $51 \%$ for predicting liver cirrhosis in comparison to F0-F3.

In this study, the sensitivity and specificity of APRI and FIB-4 were evaluated at previously studied cut-offs in expressing liver cirrhosis. For APRI, at a cut-off $>1$, the sensitivity was $59.1 \%$, the specificity was $69 \%$ and for APRI $>2$, the sensitivity was $30.5 \%$ and the specificity $89.3 \%$ for predicting cirrhosis. At a level of FIB-4 over 3.25, a sensitivity level of $52.4 \%$ and a specificity of $78.6 \%$ were determined for cirrhosis.

\section{Discussion}

Patients with HCV cirrhosis who registered SVR under DAA therapies need further monitoring in the following years as liver decompensation and hepatocellular carcinoma (HCC) could appear despite the viral clearance. In follow-up evaluations of the liver fibrosis, it could be very difficult to perform LB, especially since it has been mostly replaced by FibroTest (nowadays all other tests are being compared to it). Although it is a very useful tool, with no differences compared with LB regarding its prognostic value (15), healthcare workers are trying to find easier and less expensive methods to evaluate the degree of liver fibrosis, and especially cirrhosis.

According to the present study, both APRI and FIB-4 scores proved to be useful tools in predicting the presence of liver cirrhosis before treatment initiation as they can be rapidly calculated by screening and are less expensive than performing FibroTest. At lower levels of fibrosis (the non-cirrhotic group), neither APRI, nor FIB-4 correlated statistically with FibroTest. That is why these two scores cannot be used to differentiate between F0-F1, F1-F2 or
F2-F3. Also, at the follow-up evaluations, APRI no longer statistically correlated to the FibroTest, but FIB-4 continued to correlate with FibroTest, which shows that FIB-4 can be a useful tool for both screening for cirrhosis and for monitoring the patients after treatment with DAA (sometimes the patient is not able to pay for the FibroTest, as it is not mandatory for the patient follow-up after DAA treatment).

In a study published in 2007, Vallet-Pichard et al (14) reported that FIB-4 had an AUROC of 0.91 in identifying cirrhosis when comparing to the LB. They also performed a comparison between FIB-4 and FibroTest which showed a concordance between the two of $92.1 \%$ when FIB- 4 was $<1.45$ and $76 \%$ when FIB- 4 was $>3.25$, but for the values between 1.45 and 3.25 there was no correlation between these tests.

Another aspect observed in the present study was that between baseline and the 6-month post-EOT evaluation, there was an important decrease in the values of APRI and FIB-4, but no difference regarding the two scores between 6 months post-EOT and 12-month post-EOT could be observed. This decrease may occur as a result of the normal values of transaminases obtained after starting the DAA treatment. Similar results were published in 2017 which described a decrease of APRI and FIB-4 scores, along with decreased transient elastography (TE) results in patients who achieved SVR after DAA therapies (16).

In a study published in 2012, Tamaki et al (17) made a comparison between FIB-4 and repeated liver biopsies in evaluating the progression of liver fibrosis and concluded that using FIB-4 repeatedly, one could predict the changes in liver fibrosis every year, without having to perform LB.

Although there are many studies and systematic reviews in which the AUROCs for APRI and FIB-4 compared with LB were higher than 0.8 (classified as good to excellent) when predicting cirrhosis, we performed the AUROCs, sensitivities and specificities compared with FibroTest. Our results show lower values compared with the systematic review of Chou and Wasson (13), which reported a median AUROC for APRI of 0.84 (range, 0.54-0.97), for which an optimal cut-off of 2 had a median sensitivity of $48 \%$ (range, 17-76\%) and median specificity of $94 \%$ (range, 65-99\%). Also, the median AUROC for FIB-4 was 0.87 (range, 0.83-0.92), with an optimal cut-off of 3.25 for which the median sensitivity was $55 \%$ and the median specificity was $92 \%$.

Houot et al (18) elaborated a systematic review which concluded that for patients with $\mathrm{HCV}$, information gathered from 18 different studies, APRI had lower AUROC than FibroTest in describing different degrees of fibrosis, but without any difference regarding cirrhosis.

Cepeda et al (19) tested APRI and FIB-4 at previously validated cut-offs (only APRI had a cut-off of $>1.5$ ) in estimating severe liver stiffness by using TE. Their results for severe stiffness ( $\geq 12.3 \mathrm{kPa}$ ) show an AUROC for APRI of 0.77 (cut-off 1.5, sensitivity $61 \%$ and specificity $80 \%$ ) and for FIB-4 of 0.8 (cut-off 3.25 , sensitivity $62 \%$ and specificity $87 \%$ ). They also tried to develop new scoring systems that included FIB-4, gamma-glutamyl transferase (GGT), high-density lipoprotein (HDL), homeostatic model assessment insulin resistance (HOMA-IR) and body mass index (BMI), with enhanced accuracy for predicting cirrhosis and a simplified APRI score that added GGT, BMI and age. This new APRI score had 
higher accuracy than the classic APRI (AUROC 0.83, cut-off 0.22 , sensitivity $82 \%$ and specificity of $70 \%$ ), but all new FIB-4 models outranked APRI (the highest AUROC for FIB-4 best subset model: 0.87 , sensitivity $70 \%$ specificity $87 \%$ ).

Overall, the AUROCs for both APRI and FIB-4 determined in our study were lower than the data described in most studies, but the difference is that most studies from literature have evaluated these scores in comparison with LB (20), while our study evaluates them in comparison to FibroTest. Another explanation could be that the non-cirrhotic group might not have been as well represented as the general population of $\mathrm{HCV}$ non-cirrhotic patients.

Both APRI and FIB-4 prove to be easy, quick and inexpensive tools for screening $\mathrm{HCV}$ cirrhosis, with moderate diagnostic performance and FIB-4 can also be useful for monitoring patients post-EOT. The ideal biomarker (with very high sensitivity, specificity, specific for liver cells, reliable, useful for monitoring liver fibrosis and inexpensive) is yet to be discovered.

\section{Acknowledgements}

The follow-up FibroTests were performed with the help of BioPredictive, both for cirrhotic and non-cirrhotic patients, with help provided by Dr Mona Munteanu (BioPredictive Hepatology Research Unit; Hepatology Department, Assistance Publique-Hôpitaux de Paris, PitiéSalpêtrière Hospital, Paris, France). This study is part of 'Carol Davila' University of Medicine and Pharmacy doctoral program and will be integrated in the PhD thesis of author Anca Leuştean.

\section{Funding}

This work was partially supported by a grant of Ministry of Research and Innovation, CNCS-UEFISCDI (project no. PN-III-P4-ID-PCE-2016-0641) within PNCDI-III. This work was partially supported by a grant of Romanian Ministry of Research and Innovation, CCCDI-UEFISCDI (project no. 61PCCDI/2018 PN-III-P1-1.2-PCCDI-2017-0341) within PNCDI-III.

\section{Availability of data and materials}

The datasets used and/or analyzed during the current study are available from the corresponding author on reasonable request.

\section{Authors' contributions}

AL, CP, LN, CT and VA contributed in the conception and design of the study, data acquisition, analysis and interpretation of the data, statistical analysis, manuscript drafting, and critical revision of the manuscript for important intellectual content. All authors read and approved the final manuscript.

\section{Ethics approval and consent to participate}

The study was approved by The Ethics Committee of 'Prof. Dr. Matei Balş' National Institute for Infectious Diseases. All patients enrolled in the study gave written informed consent.

\section{Patient consent for publication}

Not applicable.

\section{Competing interests}

The authors declare that they have no competing interests.

\section{References}

1. Popescu C, Stratan L, Catană R, Leuștean A, Dragomirescu C, Badea A, Murariu C, Năstase R, Molagic V, Aramă V, et al: The efficacy of Ombitasvir-paritaprevir/ritonavir, dasabuvir and ribavirin in patients with genotype $1 \mathrm{HCV}$ compensated cirrhosis. The 12th Edition of the Scientific Days of the National Institute for Infectious Diseases "Prof. Dr. Matei Bals" and the 12th National Infectious Diseases Conference. BMC Infectious Diseases 16: 31-76, 2016, 10.1186/s12879-016-1877-4.

2. Aramă V, Leuştean A, Catană R, Stratan L, Nastase RM, Molagic V, Radulescu M, Munteanu DI, Tiliscan C, Orfanu A, et al: The efficacy of OBV/PTV/r + DSV and RBV in a Romanian cohort with genotype $1 \mathrm{HCV}$ compensated cirrhosis. Poster presentation at the 26th Annual Conference of APASL, Shanghai, China. Hepatol Int 11 (Suppl 1): S1028 (PP1765), 2017.

3. Poordad F, Hezode C, Trinh S1028R, Kowdley KV, Zeuzem S, Agarwal K, Shiffman ML, Wedemeyer H, Berg T, Yoshida EM, et al: ABT-450/r-ombitasvir and dasabuvir with ribavirin for hepatitis $\mathrm{C}$ with cirrhosis. N Engl J Med 370: 1973-1982, 2014.

4. Poynard T, Imbert-Bismut F, Munteanu M, Messous D, Myers RP, Thabut D, Ratziu V, Mercadier A, Benhamou Y and Hainque B: Overview of the diagnostic value of biochemical markers of liver fibrosis (FibroTest, HCV FibroSure) and necrosis (ActiTest) in patients with chronic hepatitis C. Comp Hepatol 3: 8, 2004.

5. Cadranel JF, Rufat P and Degos F: Practices of liver biopsy in France: Results of a prospective nationwide survey. For the group of epidemiology of the French Association for the study of the liver (AFEF). Hepatology 32: 477-481, 2000.

6. Regev A, Berho M, Jeffers LJ, Milikowski C, Molina EG, Pyrsopoulos NT, Feng ZZ, Reddy KR and Schiff ER: Sampling error and intraobserver variation in liver biopsy in patients with chronic HCV infection. Am J Gastroenterol 97: 2614-2618, 2002.

7. Bedossa $P$, Dargère $D$ and Paradis V: Sampling variability of liver fibrosis in chronic hepatitis C. Hepatology 38: 1449-1457, 2003.

8. Ferraioli G, Tinelli C, Dal Bello B, Zicchetti M, Lissandrin R, Filice G, Filice C, Above E, Barbarini G, Brunetti E, et al: Performance of liver stiffness measurements by transient elastography in chronic hepatitis. World J Gastroenterol 19: 49-56, 2013.

9. Poynard T, Munteanu M, Imbert-Bismut F, Charlotte F, Thabut D, Le Calvez S, Messous D, Thibault V, Benhamou Y, Moussalli J and Ratziu V: Prospective analysis of discordant results between biochemical markers and biopsy in patients with chronic hepatitis C. Clin Chem 50: 1344-1355, 2004.

10. Wai CT, Greenson JK, Fontana RJ, Kalbfleisch JD, Marrero JA, Conjeevaram HS and Lok AS: A simple noninvasive index can predict both significant fibrosis and cirrhosis in patients with chronic hepatitis C. Hepatology 38: 518-526, 2003.

11. Sterling RK, Lissen E, Clumeck N, Sola R, Correa MC, Montaner J, S Sulkowski M, Torriani FJ, Dieterich DT, Thomas DL, et al: Development of a simple noninvasive index to predict significant fibrosis patients with $\mathrm{HIV} / \mathrm{HCV}$ coinfection. Hepatology 43: 1317-1325, 2006.

12. Zubair I and Wajid B: Comparison of APRI, FIB-4 and fibro test in prediction of fibrosis and cirrhosis in patients with hepatitis C. 2018 15th International Bhurban Conference on Applied Sciences and Technology (IBCAST), Islamabad, pp222-227, 2018.

13. Chou R and Wasson N: Blood tests to diagnose fibrosis or cirrhosis in patients with chronic hepatitis $\mathrm{C}$ virus infection: $\mathrm{A}$ systematic review. Ann Intern Med 158: 807-820, 2013.

14. Vallet-Pichard A, Mallet V, Nalpas B, Verkarre V, Nalpas A, Dhalluin-Venier V, Fontaine H and Pol S: FIB-4: An inexpensive and accurate marker of fibrosis in HCV infection. Comparison with liver biopsy and fibrotest. Hepatology 46 : 32-36, 2007. 
15. Poynard T, Ngo Y, Perazzo H, Munteanu M, Lebray P, Moussalli J, Thabut D, Benhamou Y and Ratziu V: Prognostic value of liver fibrosis biomarkers: A meta-analysis. Gastroenterol Hepatol 7 : 445-454, 2011

16. Bachofner JA, Valli PV, Kröger A, Bergamin I, Künzler P, Baserga A, Braun D, Seifert B, Moncsek A, Fehr J, et al: Direct antiviral agent treatment of chronic hepatitis $\mathrm{C}$ results in rapid regression of transient elastography and fibrosis markers fibrosis-4 score and aspartate aminotransferase-platelet ratio index. Liver Int 37: 369-376, 2017

17. Tamaki N, Kurosaki M, Tanaka K, Suzuki Y, Hoshioka Y, Kato T, Yasui Y, Hosokawa T, Ueda K, Tsuchiya K, et al: Noninvasive estimation of fibrosis progression overtime using the FIB-4 index in chronic hepatitis C. J Viral Hepat 20: 72-76, 2013.

18. Houot M, Ngo Y, Munteanu M, Marque S and Poynard T: Systematic review with meta-analysis: Direct comparisons of biomarkers for the diagnosis of fibrosis in chronic hepatitis $\mathrm{C}$ and B. Aliment Pharmacol Ther 43: 16-29, 2016.
19. Cepeda JA, Solomon SS, Srikrishnan AK, Nandagopal P, Balakrishnan P, Kumar MS, Thomas DL, Sulkowski MS and Mehta SH: Serum fibrosis markers for the diagnosis of liver disease among people with chronic hepatitis $\mathrm{C}$ in Chennai, India. Open Forum Infect Dis 3: ofw156, 2016.

20. Amorim TG, Staub GJ, Lazzarotto C, Silva AP, Manes J, Ferronato Mda G, Shiozawa MB, Narciso-Schiavon JL, Dantas-Correa EB and Schiavon Lde L: Validation and comparison of simple noninvasive models for the prediction of liver fibrosis in chronic hepatitis C. Ann Hepatol 11: 855-861, 2012.

(i) $\odot$ This work is licensed under a Creative Commons Attribution-NonCommercial-NoDerivatives 4.0 International (CC BY-NC-ND 4.0) License. 